\title{
Perioperative management of angiotensin-converting enzyme inhibitors and/or angiotensin receptor blockers: a survey of perioperative medicine practitioners
}

\author{
Sophie L Walker ${ }^{1}$ ， Thomas EF Abbott ${ }^{1}$ ， Katherine Brown $^{1}$ ， Rupert M Pearse ${ }^{1}$, Gareth L Ackland ${ }^{\text {Corresp. }}{ }^{1}$ \\ 1 William Harvey Research Institute, QMUL, Queen Mary University of London, London, United Kingdom \\ Corresponding Author: Gareth L Ackland \\ Email address: g.ackland@qmul.ac.uk
}

Background: Angiotensin-converting enzyme inhibitors (ACEi) and angiotensin receptor blockers (ARB) are the most commonly prescribed antihypertensive medications in higher-risk surgical patients. However, there is no clinical consensus on their use in the perioperative period, in part, due to an inconsistent evidence-base. To help inform the design of a large multi-centre randomized controlled trial (ISRCTN17251494). we undertook a questionnaire-based survey exploring variability in ACEi/ARB prescribing in perioperative practice.

Methods: The online survey included perioperative scenarios to examine how consistent respondents were with their stated routine preoperative practice. Clinicians with an academic interest in perioperative medicine were primarily targeted between July and September 2017. STROBE guidelines for observational research and ANZCA Trials Group Survey Reporting recommendations were adhered to.

Results: 194 responses were received, primarily from clinicians practicing in the UK. A similar minority of respondents continue ACEi $(n=57 ; 30 \%)$ and ARBs $(n=62 ; 32 \%)$ throughout the perioperative period. However, timing of preoperative cessation was highly variable, and rarely influenced by the pharmacokinetics of individual ACE-i/ARBs. Respondents' stated routine practice was frequently misaligned with their management of common pre- and postoperative scenarios involving continuation or restarting ACE-i/ARBs.

Discussion: This survey highlights many inconsistencies amongst clinicians' practice in perioperative ACE-i/ARB management. Studies designed to reveal an enhanced understanding of perioperative mechanisms at play, coupled with randomised controlled trials, are required to rationally inform the clinical management of ACE-i/ARBs in patients most at risk of postoperative morbidity. 
1 Perioperative management of angiotensin-converting enzyme inhibitors and/or

2 angiotensin receptor blockers: a survey of perioperative medicine practitioners.

3

4 Sophie L. Walker, Tom EF Abbott, Katherine Brown, Rupert M Pearse, Gareth L Ackland.

5 William Harvey Research Institute, Queen Mary University of London, London, UK.

6

7 Correspondence to:

8 Gareth L. Ackland PhD FRCA FFICM FHEA

9 Translational Medicine and Therapeutics, William Harvey Research Institute

10 Queen Mary University of London, London EC1M 6BQ United Kingdom.

E-mail: g.ackland@qmul.ac.uk Tel: +44 2078822100

12

13

14

15 Word count: 1801

16

17 
Abstract:

Background: Angiotensin-converting enzyme inhibitors (ACEi) and angiotensin receptor blockers (ARB) are the most commonly prescribed antihypertensive medications in higher-risk surgical patients.

However, there is no clinical consensus on their use in the perioperative period, in part, due to an inconsistent evidence-base. To help inform the design of a large multi-centre randomised controlled trial (ISRCTN17251494). we undertook a questionnaire-based survey exploring variability in ACEi/ARB prescribing in perioperative practice.

Methods: The online survey included perioperative scenarios to examine how consistent respondents were with their stated routine preoperative practice. Clinicians with an academic interest in perioperative medicine were primarily targeted between July and September 2017. STROBE guidelines for observational research and ANZCA Trials Group Survey Reporting recommendations were adhered to.

Results: 194 responses were received, primarily from clinicians practicing in the UK. A similar minority of respondents continue ACEi $(n=57 ; 30 \%)$ and ARBs $(n=62 ; 32 \%)$ throughout the perioperative period. However, timing of preoperative cessation was highly variable, and rarely influenced by the pharmacokinetics of individual ACE-i/ARBs. Respondents' stated routine practice was frequently misaligned with their management of common pre- and postoperative scenarios involving continuation or restarting ACE-i/ARBs.

Discussion: This survey highlights many inconsistencies amongst clinicians' practice in perioperative ACE-i/ARB management. Studies designed to reveal an enhanced understanding of perioperative mechanisms at play, coupled with randomised controlled trials, are required to rationally inform the clinical management of ACE-i/ARBs in patients most at risk of postoperative morbidity. 


\section{Introduction}

41 Over 1.5 million high-risk patients undergo surgery in the UK every year. (Abbott et al. 2017a)

42 Angiotensin-converting enzyme inhibitors (ACEi) and angiotensin receptor blockers (ARB) are

43 the most commonly prescribed antihypertensive medications in high-risk surgical

44 patients.(Abbott et al. 2017b; Ackland et al. 2015; Pearse et al. 2014) While the benefits of

$45 \mathrm{ACEi} / \mathrm{ARBs}$ in hypertension, ischaemic heart disease, heart failure, diabetes mellitus and renal

46 disease are well-recognised,(SPRINT Research Group et al. 2015; Heart Outcomes Prevention

47 Evaluation Study et al. 2000; Yusuf et al. 2003) continuation of their use throughout the entire

48 perioperative period remains controversial.

Directly conflicting data raise significant doubts about the perioperative management of

ACEi/ARBs in noncardiac surgery. Some, but not all, early-phase observational clinical

studies(Brabant et al. 1999; Schulte et al. 2011) report association between ACEi/ARBs and

perioperative hypotension, according to a variety of definitions.(Abbott et al. 2017c; Bijker et al.

2007; Vaquero Roncero et al. 2017) However, these data contrast with the results of large

clinical database studies using objective outcome measures, which provide markedly divergent

findings in noncardiac surgery. Both failure to restart ACEi/ARB therapy after surgery,(Lee et al.

56 2015; Mudumbai et al. 2014) and not stopping ACEi/ARBs before surgery,(Roshanov et al.

57 2017) have been associated with increased incidence of postoperative mortality in both

58 noncardiac and cardiac surgery. Conversely, the Cleveland Clinic Outcomes Research group

found no association between perioperative ACEi use and the incidence of postoperative complications or mortality in $>79000$ patients undergoing non-cardiac surgery. (Turan et al. 2012) In keeping with these conflicting data, three systematic reviews conclude that the evidence surrounding perioperative ACEi/ARB use is characterised by retrospective, observational studies 
63 of low methodological quality, high risk of bias and a lack of power to explore objective

64 outcomes (most notably, postoperative morbidity).(Hollmann et al. 2018; Vaquero Roncero et al.

65 2017; Zou et al. 2016) Moreover, the failure to take into account the variable pharmacokinetic

66 characteristics of ACEi/ARBs may be an additional confounder. (Michel et al. 2013)

67 To help inform the design and conduct of a randomised controlled trial now underway

68 (ISRCTN17251494), we undertook a survey to determine the current state of practice and

69 opinions regarding perioperative $\mathrm{ACEi} / \mathrm{ARB}$ use in noncardiac surgery. Our data indicate that

70 there is widespread uncertainty regarding the perioperative use of ACEi/ARB. This reinforces

71 the need for a randomized controlled trial to inform clinical practice.

\section{Methods}

74 Study design

75 We used the PICO framework to design this online survey of practice regarding the perioperative use of ACEi/ARB, as detailed in Table 1. The study received research ethics approval (QMREC1735; Queen Mary, University of London Ethics of Research Committee) and was conducted in accordance with the principles of the Declaration of Helsinki and the Research Governance Framework. We adhered to STROBE guidelines for observational research,

80 although these do not include reporting characteristics that are specific for surveys.(von Elm et al. 2007) In addition, we also used the ANZCA Trials Group Survey Reporting List (Appendix 1) to ensure transparency and reproducibility. (Story et al. 2011)

84 Questionnaire design 
85 The survey consisted of 11 questions designed to ascertain opinions regarding perioperative

$86 \mathrm{ACEi} / \mathrm{ARB}$ use and illicit routine perioperative prescribing patterns. Questions were structured

87 using two complementary approaches (Appendix 2). Firstly, five questions had a constructed

88 response format, which were designed to directly address perioperative prescribing patterns.

89 Secondly, six questions were based on common perioperative scenarios, which aimed to examine

90 whether simple clinical guidelines for these drugs are easily generalisable. This section entailed

91 several common clinical challenges including pre- and postoperative hypertension, early

92 perioperative myocardial injury and relative postoperative hypotension [see appendix 2]. The

93 final three questions addressed respondents current grade and location of practice as well as their

94 clinical background. The survey was constructed by a writing group (KB, TA, GA) and was

95 initially piloted amongst anaesthetists at The Royal London Hospital, Barts Health NHS Trust.

96 The survey underwent external peer review and second-round piloting at the Health Services

97 Research Centre, Royal College of Anaesthetists, UK. The final version of the survey was

98 approved for clarity and feasibility by all authors.

100 Participants and survey administration

101 Participation in the survey was voluntary and responses were anonymised. Participants were

102 identified and invited via three routes: 33 attendees at the Perioperative Quality Initiative (POQI)

103 Consensus Conference on perioperative management of arterial blood pressure (2017), 161

104 principal investigators from 120 UK centres that contributed to the International Surgical

105 Outcomes Study (ISOS; appendix 3) and an open invitation to members of the Royal College of

106 Anaesthetists (issued on 26 June 2017; https:/www.rcoa.ac.uk/rcoa-presidents-news-june-2017).

107 Detailed information on the study was provided in a letter sent by e-mail explaining the goals 
108 and design of the study, specifying confidentiality and the handling of data. Formal written

109 consent was not required before participation. However, consent was implied through

110 participation. Participants answered the questions via an online survey (SurveyMonkey). Two e-

111 mail reminders were sent to optimise the response rate.

112 Statistical methods

113 Categorical data are presented as n (\%), analyzed by Fishers exact test, and presented as odds

114 ratios (95\% confidence intervals). All reported $p$ values are two-sided, with significance defined

115 by $\mathrm{p}$ values $\leq 0.05$. Statistical analyses were performed using GraphPad Prism software (La Jolla, 116 CA, USA).

\section{Results}

194 anonymised surveys were collected in total between 26 June 2017 and 31 September 2017, with a 64.4\% response rate from 125/194 POQI/ISOS investigators (Supplementary Figure 1). $183(96 \%)$ respondents practiced perioperative medicine within the United Kingdom and 163 $(84.5 \%)$ were consultants in perioperative medicine/anaesthesia

Planned preoperative prescription

A similar minority of respondents continue ACEi $(n=57 ; 30 \%)$ and ARBs $(n=62 ; 32 \%)$

127 throughout the perioperative period. Amongst the $135(70 \%)$ participants who routinely stop

128 ACEi prior to surgery, 97 (72\%) routinely recommend ACEi/ARB cessation on the day of 
129 surgery. Only $3(2 \%)$ participants base ACEi/ARB cessation on the half-life of the drug. (Figure $1301)$

131

132 Planned postoperative prescription

133 Correspondents reported that a variable time at which ACEi/ARBs were restarted after surgery

134 (Figure 2). $76(40 \%)$ and 78 (41\%) respondents would restart ACEi or ARBs within 24 hours of 135 major surgery, respectively. The practice of routinely stopping ACEi/ARBs was associated with $136>24 \mathrm{~h}$ delay in restarting ACEi/ARBs (odds ratio: 3.44 (95\% CI:1.81-6.41); $<<0.001$; Figure 3).

Management of preoperative hypertension, on the day of surgery (questions 6, 7, 8)

When faced with the scenario of an acutely hypertensive patient (mean arterial pressure

$>160 \mathrm{mmHg}$ ) immediately before surgery, respondents did not appear to consider the normally on ACEi/ARB who had stopped the drug pre-operatively, there was no association between respondents' usual pre-operative practice (continuing versus stopping ACEi/ARB) and decision to proceed to surgery on that day (OR:2.37 (95\% CI:0.85-6.2); $p=0.10$; question 7; Figure 4). Untreated hypertension was more likely to trigger postponement of surgery than hypertension in patients already prescribed ACEi/ARB (OR:1.71 (95\%CI:1.07-2.73); $\mathrm{p}=0.03$;

147 question 6; Figure 4). 
$150169(87 \%)$ respondents declined to restart ACEi/ARBs within 24h in a stable, high-risk patient

151 on chronic ACEi/ARBs if their systolic blood pressure was 90-100mmHg. For respondents who

152 advocated continuing ACEi/ARBs throughout the perioperative period, faced with this scenario

153 only $11(20 \%)$ would continue ACEi/ARBs. However, respondents who advocated continuing

$154 \mathrm{ACEi} / \mathrm{ARBs}$ throughout the perioperative period were 3 times more likely to restart the drug

155 (OR:3.17 (95\%CI: 1.26-8.24); $\mathrm{p}<0.05$; Figure 4).

\section{Management of suspected postoperative myocardial injury (questions 10, 11)}

157 Postoperative hypertension (systolic arterial pressure $>170 \mathrm{mmHg}$ ) associated with clinically

158 asymptomatic rise in plasma high-sensitivity troponin on postoperative day one prompted 110

$159(57 \%)$ of respondents to restart ACEi/ARB in patients established on this therapy (Figure 5). By

160 contrast, less than $6 \%$ commenced $\mathrm{ACEi} / \mathrm{ARB}$ in patients with a similar postoperative picture

161 who were not already receiving $\mathrm{ACEi} / \mathrm{ARBs}$, deferring to specialist advice.

\section{Discussion}

The principal finding of this study is apparent widespread uncertainty surrounding the perioperative management of ACEi/ARB, even amongst experienced clinicians with an academic interest in perioperative blood pressure control and postoperative outcomes. This may be partly due to the different indications for ACEi/ARB therapy. This mirrors the conclusions of three independent systematic reviews that were unable to provide any recommendation on 
171 perioperative management of $\mathrm{ACEi} / \mathrm{ARB}$, chiefly due to poor study design and the lack of

172 objective outcomes.(Hollmann et al. 2018; Vaquero Roncero et al. 2017; Zou et al. 2016)

173 Surgical patients with cardiac failure are at high-risk of postoperative morbidity and 174 mortality.(Abbott et al. 2016; Abbott et al. 2017c; Abbott et al. 2017d; Hammill et al. 2008;

175 Hernandez et al. 2004) Moreover, many surgical patients are deconditioned and share strikingly

176 similar cardiopulmonary physiology with cardiac failure patients.(Abbott et al. 2017b) As

177 revealed by cardiopulmonary exercise testing, many of these surgical patients have impaired left

178 ventricular function- even though they have no formal diagnosis of cardiac failure. Registry data 179 for hospitalized, cardiac failure patients shows that 30-day mortality was substantially higher in 180 those in whom ACEi/ARBs were discontinued (adjusted hazard ratio [HR $\left.\mathrm{Hdj}_{\mathrm{aj}}\right] 1.92 ; 95 \% \mathrm{CI} 1.32$ $1812.81 ; P<0.001$ ), with the readmission rate post-discharge lowest among patients continued or 182 started on therapy.(Gilstrap et al. 2017) These marked outcome differences persisted after 183 discharge, with higher one-year mortality (41.6\%) associated with discontinuation of

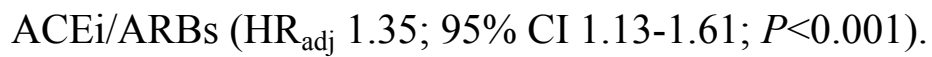
management is seldom considered by clinicians. ARBs have very different terminal half-lives, receptor binding kinetics, active metabolic components, highly variable volumes of distribution and some exhibit insurmountable antagonism, compared to ACEi.(Michel et al. 2013) Thus, a one-size-fits-all approach for perioperative ACEi/ARB management is illogical. However, studies in which perioperative ACEi/ARB therapy was stopped have failed to consider the type of $\mathrm{ACEI} / \mathrm{ARB}$, patient indication for therapy or the time point at which ACEi/ARB therapy was 192 restarted. 
Strengths of this survey, which is the largest undertaken thus far, include deliberately

194 195 196 targeting experienced clinicians (ISOS/POQI investigators) with an academic interest in perioperative blood pressure control and postoperative outcomes. A further strength was that, regardless of clinical/academic background, we probed the internal consistency of each erespondents pre- and postoperative practice by further exploring specific scenarios. This approach frequently revealed a disconnect between respondents stated routine practice and their answers to common postoperative scenarios. The survey is limited by a relatively small sample that may not be representative of global practice, which is heavily geographically biased towards the UK. The survey would be strengthened by corroborative clinical data to assess whether respondents managed patients in a real world setting in a similar manner to that reported in the survey. In accordance with ethical committee requirement for survey responses to be completely anonymised, we cannot verify the exact origin of correspondents. It is likely that more detailed scenarios including renal function may have revealed more nuanced responses.

In conclusion, this survey suggests that the clinical management of ACEi/ARB therapy is highly variable, and often internally inconsistent. This survey, and systematic reviews, highlight the need for a mechanistic randomized controlled trial, using blinded outcomes in patients most at risk of postoperative morbidity. 
211

212

213

214

215

216

217

218

219

220

221

222

223

224

225

226

227

228

229

230

231

232

233

234

235

236

237

238

239

240

241

242

243

244

245

246

247

248

249

250

251

252

253

254

255

\section{References}

Abbott TE, Ackland GL, Archbold RA, Wragg A, Kam E, Ahmad T, Khan AW, Niebrzegowska E, Rodseth RN, Devereaux PJ, and Pearse RM. 2016. Preoperative heart rate and myocardial injury after non-cardiac surgery: results of a predefined secondary analysis of the VISION study. Br J Anaesth 117:172-181. 10.1093/bja/aew182

Abbott TEF, Fowler AJ, Dobbs TD, Harrison EM, Gillies MA, and Pearse RM. 2017a. Frequency of surgical treatment and related hospital procedures in the UK: a national ecological study using hospital episode statistics. Br J Anaesth 119:249-257. 10.1093/bja/aex137

Abbott TEF, Minto G, Lee AM, Pearse RM, Ackland GL, Pom-Hr P-O, and groups Os. 2017b. Elevated preoperative heart rate is associated with cardiopulmonary and autonomic impairment in highrisk surgical patients. Br J Anaesth 119:87-94. 10.1093/bja/aex164

Abbott TEF, Pearse RM, Archbold RA, Ahmad T, Niebrzegowska E, Wragg A, Rodseth RN, Devereaux PJ, and Ackland GL. 2017c. A Prospective International Multicentre Cohort Study of Intraoperative Heart Rate and Systolic Blood Pressure and Myocardial Injury After Noncardiac Surgery: Results of the VISION Study. Anesth Analg. 10.1213/ANE.0000000000002560

Abbott TEF, Pearse RM, Archbold RA, Wragg A, Kam E, Ahmad T, Khan AW, Niebrzegowska E, Rodseth RN, Devereaux PJ, and Ackland GL. 2017d. Association between preoperative pulse pressure and perioperative myocardial injury: an international observational cohort study of patients undergoing non-cardiac surgery. Br J Anaesth 119:78-86. 10.1093/bja/aex165

Ackland GL, Iqbal S, Paredes LG, Toner A, Lyness C, Jenkins N, Bodger P, Karmali S, Whittle J, Reyes A, Singer M, Hamilton M, Cecconi M, Pearse RM, Mallett SV, Omar RZ, and group P-Os. 2015. Individualised oxygen delivery targeted haemodynamic therapy in high-risk surgical patients: a multicentre, randomised, double-blind, controlled, mechanistic trial. Lancet Respir Med 3:33-41. 10.1016/S2213-2600(14)70205-X

Bijker JB, van Klei WA, Kappen TH, van Wolfswinkel L, Moons KG, and Kalkman CJ. 2007. Incidence of intraoperative hypotension as a function of the chosen definition: literature definitions applied to a retrospective cohort using automated data collection. Anesthesiology 107:213-220. 10.1097/01.anes.0000270724.40897.8e

Brabant SM, Bertrand M, Eyraud D, Darmon PL, and Coriat P. 1999. The hemodynamic effects of anesthetic induction in vascular surgical patients chronically treated with angiotensin II receptor antagonists. Anesth Analg 89:1388-1392.

Gilstrap LG, Fonarow GC, Desai AS, Liang L, Matsouaka R, DeVore AD, Smith EE, Heidenreich P, Hernandez AF, Yancy CW, and Bhatt DL. 2017. Initiation, Continuation, or Withdrawal of Angiotensin-Converting Enzyme Inhibitors/Angiotensin Receptor Blockers and Outcomes in Patients Hospitalized With Heart Failure With Reduced Ejection Fraction. J Am Heart Assoc 6. 10.1161/JAHA.116.004675

SPRINT Research Group, Wright JT, Jr., Williamson JD, Whelton PK, Snyder JK, Sink KM, Rocco MV, Reboussin DM, Rahman M, Oparil S, Lewis CE, Kimmel PL, Johnson KC, Goff DC, Jr., Fine LJ, Cutler JA, Cushman WC, Cheung AK, and Ambrosius WT. 2015. A Randomized Trial of Intensive versus Standard Blood-Pressure Control. N Engl J Med 373:2103-2116. 10.1056/NEJMoa1511939

Hammill BG, Curtis LH, nett-Guerrero E, O'Connor CM, Jollis JG, Schulman KA, and Hernandez AF. 2008. Impact of heart failure on patients undergoing major noncardiac surgery. Anesthesiology 108:559-567.

Heart Outcomes Prevention Evaluation Study I, Yusuf S, Sleight P, Pogue J, Bosch J, Davies R, and Dagenais G. 2000. Effects of an angiotensin-converting-enzyme inhibitor, ramipril, on 
cardiovascular events in high-risk patients. N Engl J Med 342:145-153. 10.1056/NEJM200001203420301

Hernandez AF, Whellan DJ, Stroud S, Sun JL, O'Connor CM, and Jollis JG. 2004. Outcomes in heart failure patients after major noncardiac surgery. J Am Coll Cardiol 44:1446-1453. S0735-1097(04)013592 [pii]

10.1016/j.jacc.2004.06.059

Hollmann C, Fernandes NL, and Biccard BM. 2018. A Systematic Review of Outcomes Associated With Withholding or Continuing Angiotensin-Converting Enzyme Inhibitors and Angiotensin Receptor Blockers Before Noncardiac Surgery. Anesth Analg. 10.1213/ANE.0000000000002837

Lee SM, Takemoto S, and Wallace AW. 2015. Association between Withholding Angiotensin Receptor Blockers in the Early Postoperative Period and 30-day Mortality: A Cohort Study of the Veterans Affairs Healthcare System. Anesthesiology 123:288-306. 10.1097/ALN.0000000000000739

Michel MC, Foster C, Brunner HR, and Liu L. 2013. A systematic comparison of the properties of clinically used angiotensin II type 1 receptor antagonists. Pharmacol Rev 65:809-848. 10.1124/pr.112.007278

Mudumbai SC, Takemoto S, Cason BA, Au S, Upadhyay A, and Wallace AW. 2014. Thirty-day mortality risk associated with the postoperative nonresumption of angiotensin-converting enzyme inhibitors: a retrospective study of the Veterans Affairs Healthcare System. J Hosp Med 9:289296. 10.1002/jhm.2182

Pearse RM, Harrison DA, MacDonald N, Gillies MA, Blunt M, Ackland G, Grocott MP, Ahern A, Griggs K, Scott R, Hinds C, and Rowan K. 2014. Effect of a Perioperative, Cardiac Output-Guided Hemodynamic Therapy Algorithm on Outcomes Following Major Gastrointestinal Surgery: A Randomized Clinical Trial and Systematic Review. JAMA. 10.1001/jama.2014.5305

1873985 [pii]

Roshanov PS, Rochwerg B, Patel A, Salehian O, Duceppe E, Belley-Cote EP, Guyatt GH, Sessler DI, Le Manach Y, Borges FK, Tandon V, Worster A, Thompson A, Koshy M, Devereaux B, Spencer FA, Sanders RD, Sloan EN, Morley EE, Paul J, Raymer KE, Punthakee Z, and Devereaux PJ. 2017. Withholding versus Continuing Angiotensin-converting Enzyme Inhibitors or Angiotensin II Receptor Blockers before Noncardiac Surgery: An Analysis of the Vascular events In noncardiac Surgery patlents cOhort evaluatioN Prospective Cohort. Anesthesiology 126:16-27. 10.1097/ALN.0000000000001404

Schulte E, Ziegler D, Philippi-Hohne C, Kaczmarczyk G, and Boemke W. 2011. Angiotensin-converting enzyme inhibition and blood pressure response during total intravenous anaesthesia for minor surgery. Acta Anaesthesiol Scand 55:435-443. 10.1111/j.1399-6576.2011.02409.x

Story DA, Gin V, na Ranong V, Poustie S, Jones D, and Group AT. 2011. Inconsistent survey reporting in anesthesia journals. Anesth Analg 113:591-595. 10.1213/ANE.0b013e3182264aaf

Turan A, You J, Shiba A, Kurz A, Saager L, and Sessler DI. 2012. Angiotensin converting enzyme inhibitors are not associated with respiratory complications or mortality after noncardiac surgery. Anesth Analg 114:552-560. 10.1213/ANE.0b013e318241f6af

Vaquero Roncero LM, Sanchez Poveda D, Valdunciel Garcia JJ, Sanchez Barrado ME, and Calvo Vecino JM. 2017. Perioperative use of angiotensin-converting-enzyme inhibitors and angiotensin receptor antagonists. J Clin Anesth 40:91-98. 10.1016/j.jclinane.2017.04.018

von Elm E, Altman DG, Egger M, Pocock SJ, Gotzsche PC, Vandenbroucke JP, and Initiative S. 2007. Strengthening the Reporting of Observational Studies in Epidemiology (STROBE) statement: guidelines for reporting observational studies. BMJ 335:806-808. 10.1136/bmj.39335.541782.AD

Yusuf S, Pfeffer MA, Swedberg K, Granger CB, Held P, McMurray JJ, Michelson EL, Olofsson B, Ostergren $J$, Investigators $C$, and Committees. 2003. Effects of candesartan in patients with chronic heart 
303 failure and preserved left-ventricular ejection fraction: the CHARM-Preserved Trial. Lancet 304 362:777-781. 10.1016/S0140-6736(03)14285-7

305 Zou Z, Yuan HB, Yang B, Xu F, Chen XY, Liu GJ, and Shi XY. 2016. Perioperative angiotensin-converting

306 enzyme inhibitors or angiotensin II type 1 receptor blockers for preventing mortality and

307 morbidity in adults. Cochrane Database Syst Rev:CD009210. 10.1002/14651858.CD009210.pub2

308

309

310 


\section{Table $\mathbf{1}$ (on next page)}

PICO model to frame questions for survey. 
1 Table 1. PICO model to frame questions for survey.

2

\begin{tabular}{|l|l|}
\hline PATIENT: & $\begin{array}{l}\text { Patients undergoing non-cardiac surgery, requiring ACE- } \\
\text { inhibitor and/or angiotensin receptor blocking therapy for } \\
\text { cardiometabolic and/or renal disease. }\end{array}$ \\
\hline INTERVENTION: & $\begin{array}{l}\text { Withdrawal of ACE-inhibitor and/or angiotensin receptor } \\
\text { blockade therapy }\end{array}$ \\
\hline COMPARISON: & $\begin{array}{l}\text { Continuation of ACE-inhibitor and/or angiotensin receptor } \\
\text { blockade therapy. }\end{array}$ \\
\hline OUTCOME: & $\begin{array}{l}\text { Identify perioperative practice under different common } \\
\text { clinical scenarios. }\end{array}$ \\
\hline
\end{tabular}




\section{Figure 1 (on next page)}

Routine pre-operative practice for ACEi and ARB. 


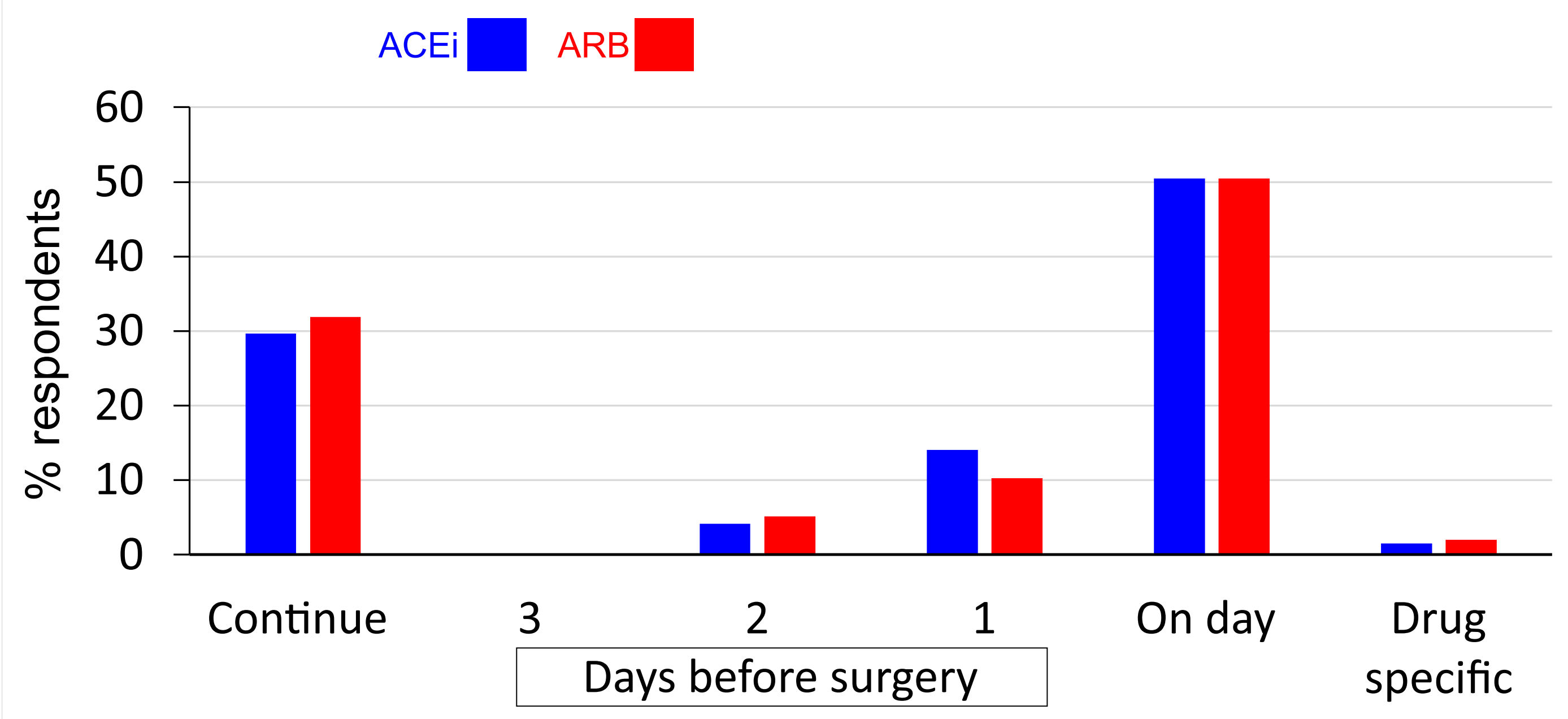


Figure 2 (on next page)

Routine post-operative practice for ACEi and ARB use. 


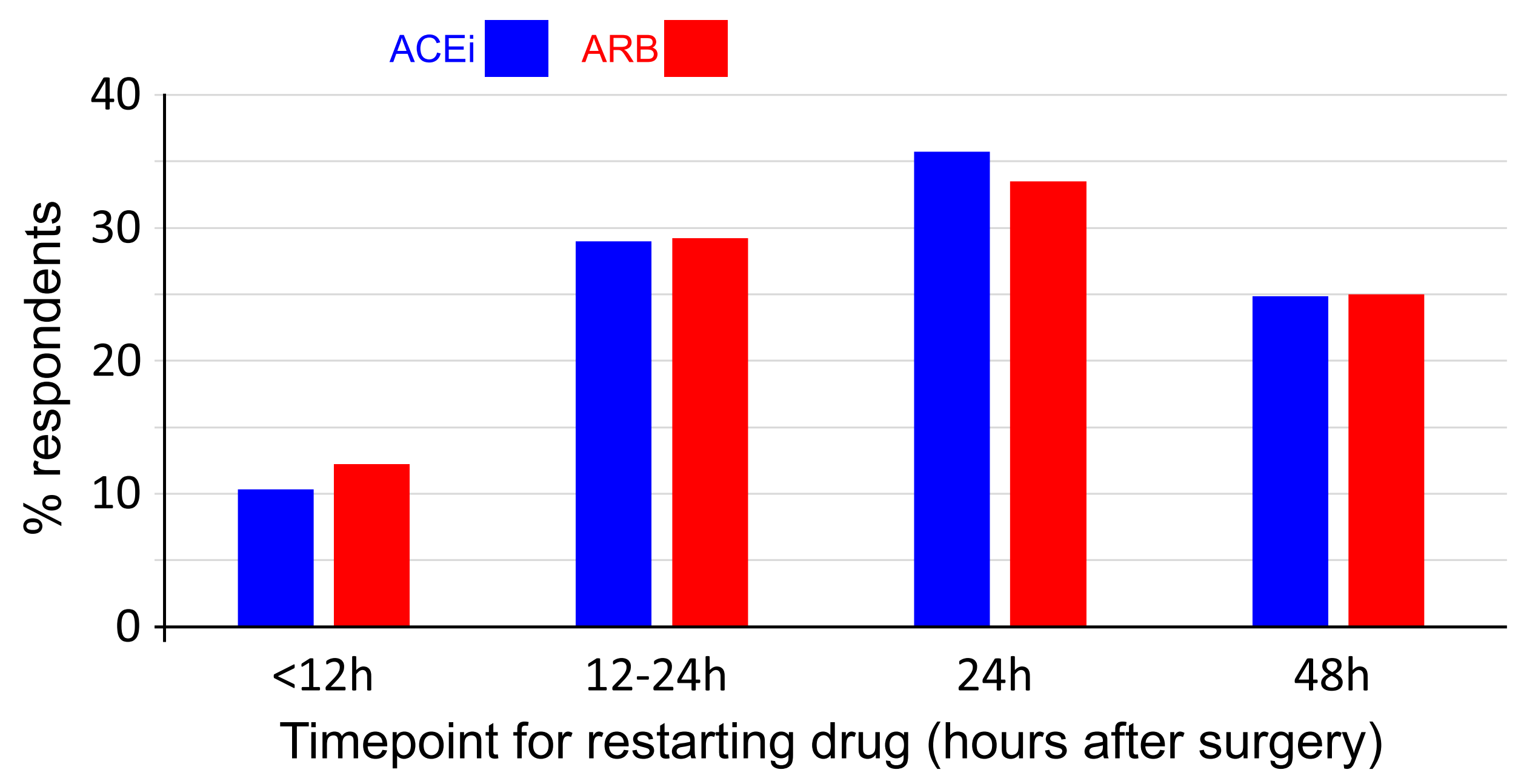




\section{Figure 3}

Association between planned pre- and post-operative practice.

Routinely stopping ACEi/ARBs in the pre-operative setting was associated with a $>24 \mathrm{hr}$ delay in restarting the drug (odds ratio: 3.44 (95\% Cl:1.81-6.41); $p<0.001)$.

\section{Post-operative Practice}

Of those that would stop...

\section{Post-operative Practice} Of those that would continue...

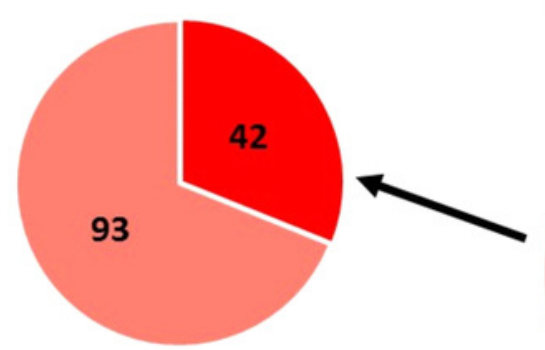

- Restart with 24 hours

- Restart $>24$ hours
Pre-operative Practice
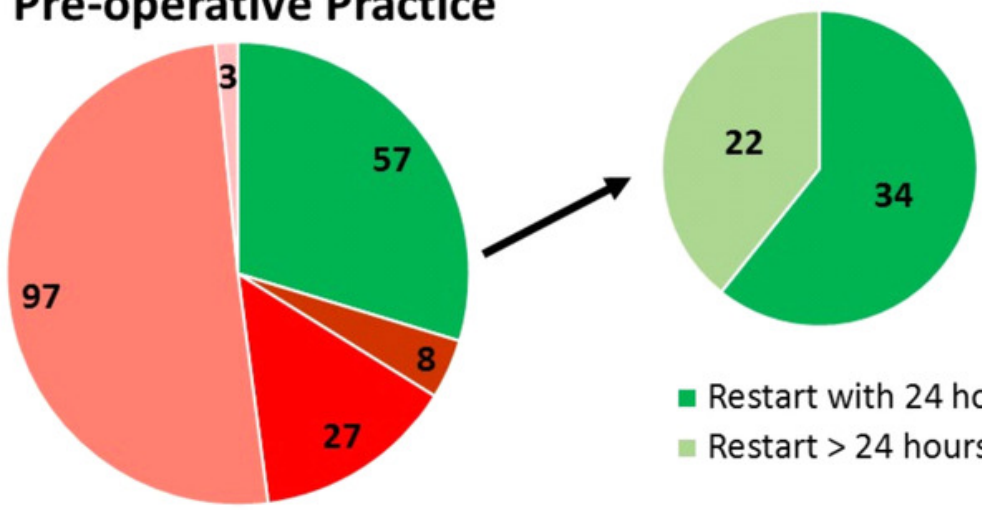

- Restart with 24 hours

nestart $>24$ hours

- Continue ACE inhibitor

- Stop 2 days before surgery

- Stop on day before surgery

= Stop on day of surgery

In Stop (duration of stopping depending on the drug) 
Figure 4

Clinical scenarios: survey questions 6-9.

$A$ of those who would stop...

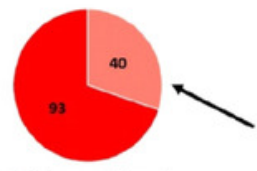

" Postpone = Proceed

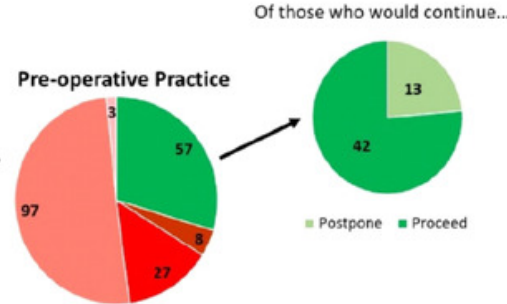

Continue drug

Stop 2 days before surgery

Stop on day before surgery

- Stop on day of surgery

Stop [depending on drug

C

Of those who would stop..

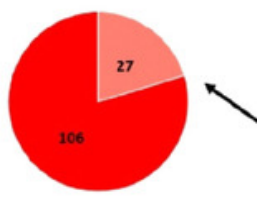

= Postpone = Proceed
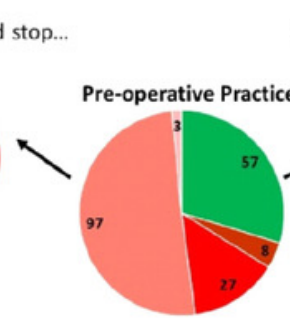

Of those who would continue...

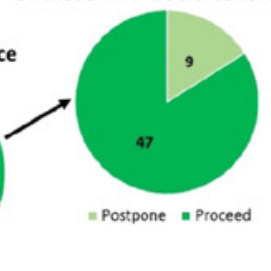

B

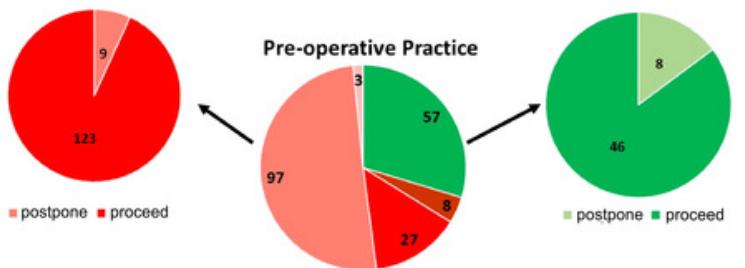

Continue drug

Stop 2 days before surgery

Stop on day before surgery

Stop on day of surgery

$\mathrm{D}$ Of those who would stop...

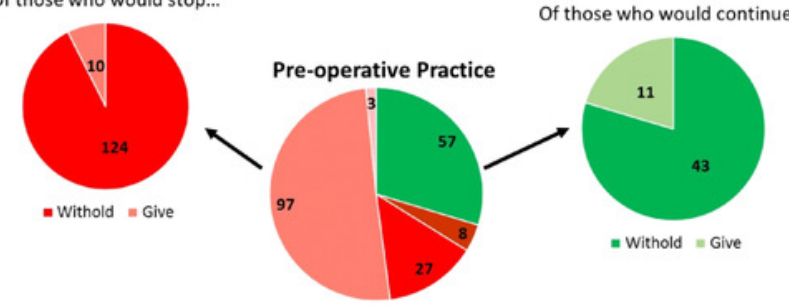

Continue drug

- Stop 2 days before surgery - Stop on day before surgery Stop on day of surgery 


\section{Figure 5}

Impact of postoperative myocardial injury on ACEi/ARB use.

An asymptomatic rise in troponin post-operatively would prompt $110(57 \%)$ to restart ACEi/ARBs in those already established on this therapy. In those patients not already receiving ACEi/ARBs, a similar asymptomatic troponin rise prompts only $6 \%$ to commence the drug deferring to specialist advice.

A

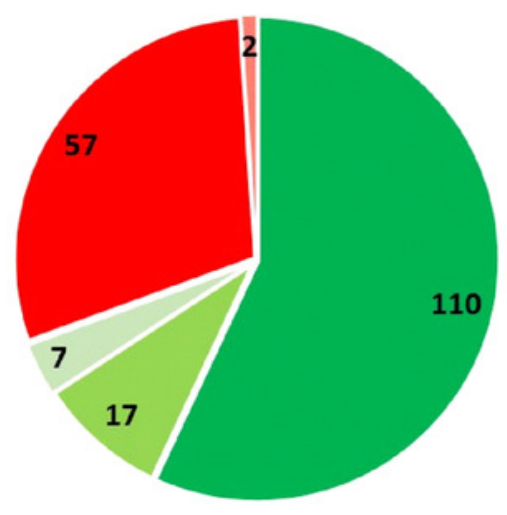

- Re-start their ACE-i/ARB

- Treat acutely to reduce systolic BP to $<160 \mathrm{mmHg}$

= Start other (longer-acting) anti-hypertensives

- Refer for medical advice

n Continue without intervention but monitor
B

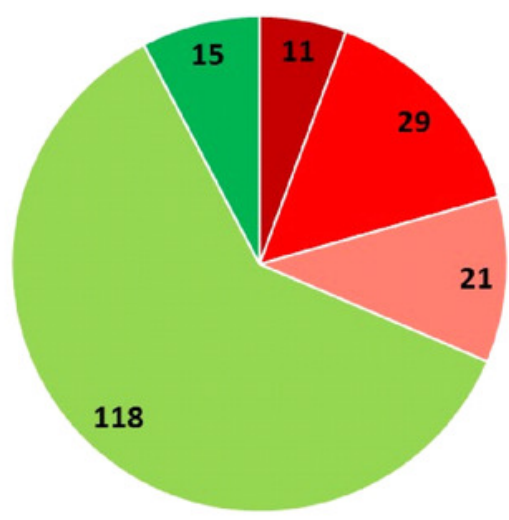

- Start an ACE-i or ARB

- Treat acutely to reduce systolic BP to $<160 \mathrm{mmHg}$

= Start long-acting anti-hypertensives

nefer for medical advice

- Continue without intervention but monitor 